\title{
STIMULATING URBAN GOMPETITIVENESS BY NETWORKING. PART II - CASE STUDY: ROMANIIAN GITIES INVOLVED IN URBACT PROJECTS
}

\author{
Ștefana VARVARI ${ }^{a *}$, Dana BAKO ${ }^{\mathrm{b}}$ \\ a),b) Babeș-Bolyai University, Faculty of Economics and Business Administration, \\ Cluj-Napoca, Romania
}

Please cite this article as:

Article History:

Varvari, S.. and Bako, D. 2019. Stimulating urban Received: 12 November 2018 competitiveness by networking. Part II - Case study: Accepted: 18 March 2019 Romanian cities involved in URBACT projects. Review of Economic Studies and Research Virgil Madgearu, 12(1), pp.97-128.

doi: 10.24193/RVM.2019.12.35.

Abstract: There are major differences regarding the supply of "attractiveness" factors by different cities in Europe. One way in which less attractive cities could become more desirable is through the cooperation with other, more competitive cities of the EU, by exchanging good practices and knowledge, working together for finding best solutions for common problems. The first part of the paper has drawn some conclusions regarding the main results and improvements brought by the three URBACT programmes. In this second part, the paper analyses the participation of Romanian cities in these programmes. Romanian cities were partners in many of the networks created within URBACT, some of them being more involved than others (eg. Alba Iulia). Also, the networks with Romanian cities focused mostly on governance and economy, showing that these cities need support in building their capacities and improving their economies.

Key words: integrated urban development; urban attractiveness; urban networks; knowledge transfer JEL Classification: $O 18$; $R 11$

(C) 2019 Alma Mater Publishing House. All rights reserved.

* Corresponding author. E-mail address: stefana.varvari@econ.ubbcluj.ro. 
Review of Economic Studies and Research Virgil Madgearu, 2019, 12(1)

\section{References}

Official documents and webpage of the URBACT programme:

1. European Commission - URBACT, 2007a. The URBACT programme 2002-2006. BILAN. [pdf] European Commission. Available at $<$ http://urbact.eu/sites/default/files/import/general_library/ URBACT_GB_01.pdf> [Accessed April 2018].

2. European Commission - URBACT, 2007b. The URBACT programme. Support for Cities. Operational report on the expert's missions in the framework of the support for cities - initiative. [pdf] European Commission. Available at <http://urbact.eu/sites/ default/files/sfcurbact.en_01.pdf> [Accessed April 2018].

3. European Commission - URBACT, 2017a. The URBACT II Programme. FINAL Implementation Report, CCI 2007 CB 163 PO 048. [pdf] European Commission. Available at <http://urbact.eu/ sites/default/files/fir_final_draft.pdf> [Accessed April 2018].

4. European Commission - URBACT, 2017b. The URBACT III Programme. 2016 Annual Implementation Report, CCI 2014 TC 16 RFIR 003. [pdf] European Commission. Available at $<$ http://urbact. eu/sites/default/files/air_2016_final.pdf> [Accessed April 2018].

5. European Commission, 2018a. URBACT programme. [online] Available at <http://urbact.eu > [Accessed April 2018].

6. European Commission, 2018b. URBACT networks. [online] Available at <http://urbact.eu/all-networks > [Accessed April 2018].

Official documents (baseline studies, thematic analyses, progress and final reports) on webpages of the URBACT projects:

7. European Commission - URBACT, 2018c. BOOSTINNO Project. [online] Available at <http://urbact.eu/boostinno> [Accessed October 2018].

8. European Commission - URBACT, 2018d. Building Healthy Communities Project. [online] Available at <http://urbact.eu/ building-healthy-communities $>$ [Accessed October 2018].

9. European Commission - URBACT, 2018e. CityLogo Project. [online] Available at <http://urbact.eu/citylogo $>$ [Accessed October 2018].

10. European Commission - URBACT, 2018f. CoNet Project. [online] Available at <http://urbact.eu/conet $>$ [Accessed October 2018].

11. European Commission - URBACT, 2018g. ESIMeC Project. [online] Available at <http://urbact.eu/esimec $>$ [Accessed October 2018]. 
VARVARI AND BAKo, STIMULATING URban COMPETITIVENESS By NETWORKING...

12. European Commission - URBACT, 2018h. ESIMeC II Project. [online] Available at <http://urbact.eu/esimec-ii > [Accessed October 2018].

13. European Commission - URBACT, 2018i. EVUE Project. [online] Available at <http://urbact.eu/evue > [Accessed October 2018].

14. European Commission - URBACT, 2018j. EVUE II Project. [online] Available at <http://urbact.eu/evue-ii $>$ [Accessed October 2018].

15. European Commission - URBACT, 2018k. Freight TAILS Project. [online] Available at <http://urbact.eu/freight-tails> [Accessed October 2018].

16. European Commission - URBACT, 2018l. Gastronomic Cities Project. [online] Available at <http://urbact.eu/gastronomic-cities > [Accessed October 2018].

17. European Commission - URBACT, 2018m. INT-HERIT Project. [online] Available at <http://urbact.eu/int-herit $>$ [Accessed October 2018].

18. European Commission - URBACT, 2018n. Interactive Cities Project. [online] Available at <http://urbact.eu/interactive-cities> [Accessed October 2018].

19. European Commission - URBACT, 20180. JobTown 2 Project. [online] Available at <http://urbact.eu/jobtown-2> [Accessed October 2018].

20. European Commission - URBACT, 2018p. LUMASEC Project. [online] Available at <http://urbact.eu/lumasec $>$ [Accessed October 2018].

21. European Commission - URBACT, 2018q. Nodus Project. [online] Available at <http://urbact.eu/nodus $>$ [Accessed October 2018].

22. European Commission - URBACT, 2018r. RetailLink Project. [online] Available at <http://urbact.eu/retailink $>$ [Accessed October 2018].

23. European Commission - URBACT, 2018s. SmartImpact Project. [online] Available at <http://urbact.eu/smartimpact> [Accessed October 2018].

24. European Commission - URBACT, 2018t. sub>urban Project. [online] Available at <http://urbact.eu/sub.urban> [Accessed October 2018].

25. European Commission - URBACT, 2018u. TUTUR Project. [online] Available at <http://urbact.eu/tutur $>$ [Accessed October 2018]. 
26. European Commission - URBACT, 2018v. URBACT Markets Project. [online] Available at <http://urbact.eu/urbact-markets> [Accessed October 2018].

27. European Commission - URBACT, 2018w. USEAct Project. [online] Available at <http://urbact.eu/useact-urban-sustainableenvironmental-actions $>$ [Accessed October 2018]. 